\title{
Reseña - empírica del artículo: Control del estrés laboral en los profesores mediante educación emocional
}

\author{
María Fernanda Cabellero Loazada ${ }^{1}$ \\ Mónica Fernanda Chica² \\ Julio Cesar Montoya R. ${ }^{3}$
}

\section{Resumen}

El presente documento es una reseña sobre el articulo empírico “Control del Estrés Laboral en los profesores mediante Educación Emocional” ; para ello se realizó una identificación detallada de los principales aspectos tratados en la Introducción, problema, objetivo e hipótesis de investigación, método, resultados, los aspectos que los autores sugieren para las nuevas investigaciones, alcances, limitaciones y aportes. De manera general se observó que en todas las variables estudiadas se aprecian cambios en sentido positivo que frente a la intervención realizada, afirma que la ejecución de este tipo de procedimientos dan buenos resultados, por lo tanto se promueven en varios contextos y poblaciones para generar cambios que permitan el control y el manejo del estrés laboral. Una limitación encontrada en la intervención fue no presentar el proceso de descripción que se realiza durante la intervención de las técnicas de mejoramiento. Se hace necesario reconsiderar investigaciones de tipo cualitativo-

\footnotetext{
1 Psicóloga, Magíster en Psicología, estudiante de Doctorado en Psicología, docente Universidad Nacional Abierta y a Distancia. Grupo de investigación: Subjetividades y sujetos colectivos. maria.caballero@unad.edu.co

${ }^{2}$ Psicología, Especialista en Pedagogía para el Desarrollo del Aprendizaje Autónomo y Magíster en Administración de las Organizaciones. Docente Universidad Nacional Abierta y a Distancia - UNAD monica.chica@unad.edu.co

${ }^{3}$ Administrador de Empresas, Especialista en Pedagogía para el Desarrollo del Aprendizaje Autónomo, Maestría en Administración de Empresas. Investigador asociado del grupo de investigación llama. Docente de la Universidad Nacional Abierta y a Distancia - UNAD. julio.montoya@unad.edu.co
} 
cuantitativo o mixtas. La posibilidad de efectuar una intervención con un seguimiento sistemático constituye un factor clave para el resultado de este estudio.

Tipo de Documento: Artículo de revista.

Palabras Clave: Educación emocional; estrés docente; intervención en la escuela

\section{Pincipales aspectos tratados en el apartado de Introducción}

El artículo de Salmurri \& Skoknic (2003) plantea los siguientes aspectos:

- Los numerosos estudios efectuados, comienzan por constatar la presencia, entre los maestros de un índice alto o extremadamente alto de estrés laboral.

- Las escuelas están experimentando dificultades crecientes en sus esfuerzos para generar un ambiente favorable al aprendizaje y la enseñanza; esto crea dificultades severas al trabajo de los profesores, debido a que ha aumentado la agresividad y la falta de respeto q incide en el trabajo de los docentes. Las formas hasta ahora utilizada no han traído un real mejoramiento, sólo conducen a la exclusión y al castigo de los alumnos para controlar las manifestaciones de violencia, lo cual no ha sido de ninguna manera eficaz.

- La utilidad del aprendizaje, entrenamiento y práctica de diversas habilidades emocionales, sociales, cognitivas y conductuales, ha tenido efecto en varias poblaciones y ha generado cambios positivos. Es por esta razón que se propone metodologías que faciliten controlar los niveles de tensión existentes en el ambiente escolar 


\section{Problema y objetivo de Investigación:}

El objetivo del artículo es facilitar la mejora de los recursos conductuales, cognitivos, emocionales y de interacción social de los profesores, a fin de contribuir al auto control de su estrés laboral.

\section{Hipótesis de investigación:}

La hipótesis de Salmurri \& Skoknic (2003) plantea lo siguiente: si se mejora los recursos (conductuales, cognitivos y emocionales) técnicamente planteados, se va a mejorar la interacción social de los profesores superando así el estrés laboral

\section{METODO}

Tipo de investigación: Cuantitativa, por procesos cuasi-experimentales.

\section{Población-Muestra-Sujetos-Participantes:}

50 profesores, clasificados en dos grupos 25 del grupo bajo estudio (o experimental) y 25 del grupo de comparación. Los dos grupos han sido tomados de Profesores de Enseñanza Básica que laboran en una escuela pública.

\section{Tecnicas de recolección de la información:}

Para la recolección de datos se utilizaron los siguientes instrumentos (estos se utilizaron antes y después de la intervención):

- S.C.L.-90-R: Cuestionario de Psicopatología revisado de Derogatis.

- Escala de Asertividad de Gambril y Richey. 
- E.P.Q.-A: Cuestionario de Personalidad de Eysenck y Sybil.

- T.C.I.: Cuestionario de Temperamento y Carácter.

- Escalas de Autodirección y de Cooperaciónde Cloninger.

Un componente de la intervención consistió en el aprendizaje y la práctica, realizada en veinticuatro talleres de hora y media de duración cada uno, donde se abordó los siguientes aspectos:

- Técnicas de respiración profunda y relajación según el método de entrenamiento autógeno de Shultz.

- Técnicas cognitivas: práctica de pensamiento positivo, auto-observación, introspección, detención del pensamiento.

- Habilidades y técnicas conductuales: Priorización de objetivos realistas

- Habilidades de interacción: asertividad,

\section{Técnicas de análisis de la información}

La técnica de análisis de información en esta investigación se realizó haciendo contraste con los instrumentos utilizados antes de comenzar la intervención y después de finalizar la misma, para mirar las puntuaciones y así detectar si hubo cambios o no, en el mejoramiento del estrés laboral en los profesores.

\section{Procedimiento:}

Al principio de la intervención se realizó una evaluación de su autoestima, así como de su autocontrol, estilo cognitivo positivo y de sus relaciones con las otras personas, con una escala de evaluación de 0 a 10. 
Todo el proceso de intervención se realizó en 4 etapas:

En la primera etapa, durante el Curso 1996-97 se efectuó la selección de las escuelas y se formalizó administrativamente el proceso que permitiría este estudio, el cual fue además presentado a los claustros de cada escuela para su autorización.

La segunda etapa fue en el Curso 1997-98, donde se realizaron, veinticuatro sesiones de entrenamiento divididos en dos grupos de 12 y 13 sujetos (12 sesiones por grupo con una duración de hora y media por sesión).

La tercera etapa fue en el Curso 1998-99 donde se destinaron otras veinte sesiones, conjuntamente con los profesores, a fin de establecer el sistema más adecuado para introducir este programa en el alumnado, tanto en cuanto a conceptos como a prácticas. a lo largo del curso. Al mismo tiempo, se realizaron tres sesiones de información a los padres de los alumnos sobre este estudio

La última etapa fue durante los Cursos 1999-00 y 2000-01 donde se continuó el entrenamiento del alumnado por parte de sus profesores; sin embargo, durante el curso 2000-01 se han mantenido 24 sesiones más.

La intervención realizada fue evaluada en cada etapa, mediante diversos instrumentos, al finalizar las sesiones de entrenamiento, se procedía a una "valoración global del componente entrenamiento"

\section{Resultados:}


En este proceso se consideran los datos provenientes de los 25 participantes iniciales y los 18 finales, dado que en el transcurso de los años influyó en una pérdida muestral considerada normal dada la extensión del estudio, producto de situaciones administrativas o personales no vinculadas a la intervención.

Dentro de las variables estudiadas se presentaron los siguientes resultados:

- La variable neuroticismo evaluada mediante el cuestionario de personalidad EPQ de Eysenck, muestran una tendencia a la mejora del grupo experimental a lo largo de las cuatro evaluaciones.

- Se observa que la extraversión en el grupo experimental presenta puntuaciones que indican mayor grado de extroversión. El comportamiento de las puntuaciones del grupo de comparación es ligeramente más estable, sin embargo, estas son siempre más elevadas que las del grupo experimental.

- En la variable Autocontrol, el índice de autodirección del Cuestionario de Temperamento y Carácter (TCI) de Cloninger (1987), muestra en la gráfica una tendencia a la mejora en el grupo experimental, después de la intervención.

- En la sintomatología psicopatológica la disminución de la sintomatología es significativa entre la primera y segunda evaluación e igualmente existe diferencia estadísticamente significativa entre la primera y tercera evaluación. Dicha mejora se estabiliza en la cuarta evaluación; sin embargo, existe gran variabilidad entre los sujetos de la muestra, motivo por el cual los datos no alcanzan significación estadística.

- El total de síntomas positivos de "distress evoluciona en el grupo experimental 
- La sintomatología de Somatización presenta que las puntuaciones del grupo experimental son significativamente menores a las del grupo de comparación. Por otro lado, la presencia de somatizaciones disminuyó de forma significativa en el grupo experimental.

- En la Sintomatología de Obsesión-Compulsión la sub escala de síntomas obsesivos de este instrumento, muestra un descenso significativo en el grupo experimental entre la primera y la segunda evaluación

- En cuanto a la sensibilidad Interpersonal, la variable Depresión evaluada dentro de la subescala de Depresión del Cuestionario SCL-90-R presenta una tendencia a la mejora en el grupo experimental a lo largo de la aplicación del programa.

- La variable Ansiedad informa de mejoras significativas (disminución de síntomas ansiosos) en el grupo experimental a lo largo de toda la intervención.

- La variable Hostilidad presenta las puntuaciones en esta subescala del Cuestionario de SCL90-R indican puntuaciones menores en el grupo experimental, sobre todo en dos momentos de la intervención.

- La variable Ansiedad fóbica muestra que la presencia de síntomas de ansiedad se mantiene sin cambios a lo largo de la intervención.

- La variable Ideación paranoide muestra que la presencia de síntomas de ansiedad se mantiene sin cambios a lo largo de la intervención. 
De manera general se puede ver que en todas las variables se aprecian cambios en sentido positivo, es decir, de mejora. En relación al comportamiento asertivo, tanto el "grado de ansiedad", como la "probabilidad de respuesta" de la escala de asertividad, las reducciones observadas son estadísticamente significativas después de la intervención directa con el profesorado y continúan declinando el año siguiente.

La autodirección, es decir, el autocontrol puede llevar a sugerir que este cambio puede estar vinculado a una mejora de la dinámica del entorno laboral y que ésta se mantiene a lo largo del tiempo, una vez conseguida. Esta variable se mantiene estable en las últimas mediciones, lo que indicaría una tendencia de los profesores a establecer relaciones interpersonales más empáticas y solidarias, con interesantes implicaciones en el ámbito escolar.

Solamente en una escala, la de "Ansiedad fóbica", no hay cambios relevantes.

\section{Que sugieren los autores para nuevas investigaciones}

Los autores al mostrar resultados positivos frente a la intervención realizada, dejan entrever que la ejecución de este tipo de procedimientos dan buenos resultados, por lo tanto se promueven en varios contextos y poblaciones para generar cambios que permitan el control y el manejo del estrés laboral.

\section{Alcances y limitaciones del estudio}

Una limitación grande de la intervención presentada por Salmurri \& Skoknic (2003) es no presentar el proceso de descripción que se realiza durante la intervención de las técnicas de mejoramiento. Es importante resaltar la descripción, puesto que es ese paso el que genera cierta inquietud del cómo llegaron a los resultados. 
Con lo anteriormente planteado, se hace necesario reconsiderar investigaciones de tipo cualitativocuantitativo o mixtas, es decir que combinen las dos metodologías, lo cual nos indica que en la investigación aplicada a la educación, como en general en las investigaciones en el campo social, puede ser provechoso realizar la investigación de manera mixta para realizar un análisis más detallado y profundo que incluya la riqueza narrativa y descriptiva.

Por otra parte, la investigación plantea un modelo muy conductual que se enfoca mucho en el individuo, sería pertinente que incluyera el entorno social. Sin embargo, el mismo hecho de ser una investigación cuantitativa permite presentar claramente y de manera numérica los alcances y logros que tuvo la intervención.

\section{Aportes:}

El aprendizaje y el entrenamiento en las técnicas y habilidades del programa para

profesores parece influir en el comportamiento de las variables estudiadas, lo cual lo hace un modelo útil de ser retomado y tenido en cuenta en la intervención con docentes.

Uno de los principales objetivos iniciales de este estudio ha sido comprobar los beneficios que en el terreno de la salud psicológica pudieran derivarse de la aplicación de una intervención como la descrita, tanto en adultos, como en la población infantil, por lo tanto esto abre el panorama al campo del bienestar y la calidad de vida.

La posibilidad de efectuar una intervención con un seguimiento sistemático constituye un factor clave para el resultado de este estudio. Ello parece confirmar la necesidad que el psicólogo efectúe intervenciones sistemáticas y estructuradas para el mantenimiento de las mejoras que logre, 
estableciendo la consistencia de estos cambios y detectando relaciones de causalidad que faciliten el trabajo profesional futuro.

\section{BIBLIOGRAFIA}

Salmurri, F \& Skoknic V (2003) Control del Estrés Laboral en los profesores mediante Educación Emocional. Revista de Psicología de la Universidad de Chile, Vol XII (1) 37-64 